\title{
Aggravation of hyperthyroidism after heterologous prime-boost immunization with inactivated and adenovirus-vectored SARS-CoV-2 vaccine in a patient with Graves' disease
}

\author{
Chutintorn Sriphrapradang $\mathbb{D}^{1}$
}

Received: 6 September 2021 / Accepted: 11 September 2021 / Published online: 17 September 2021

(c) The Author(s), under exclusive licence to Springer Science+Business Media, LLC, part of Springer Nature 2021

Vera-Lastra et al. [1] and Zettinig et al. [2] recently reported four cases of Graves' disease that developed after severe acute respiratory syndrome coronavirus 2 (SARSCov-2) BNT162b mRNA vaccination (Pfizer-BioNTech). I herein report a case with worsened Graves' hyperthyroidism after heterologous prime-boost immunization with inactivated and adenovirus-vectored SARS-CoV-2 vaccine.

A 30-year-old female physician was diagnosed with Graves' disease in October 2018 with a TSH receptor antibody (TRAb) level at 36.15 IU/L (N, 0-1.75). Her father, mother, and brother also had a history of Graves' disease. Hyperthyroidism was adequately controlled by methimazole $2.5 \mathrm{mg}$ per day. The summary of thyroid function tests (TFTs) was shown in Fig. 1. She had been vaccinated with two doses of inactivated whole-virus SARS-CoV-2 vaccine CoronaVac (Sinovac Biotech, China) on 7 April and 26 April 2021. Due to the B.1.617.2 (delta) variant pandemic in Thailand, she received a booster vaccine with a replication-deficient chimpanzee adenovirusvectored vaccine ChAdOx1 nCoV-19 (AZD1222, OxfordAstraZeneca) on 19 July 2021. Four days after vaccination, she noticed palpitations and needed propranolol to control her symptoms. She lost weight of $2 \mathrm{~kg}$ despite an increased appetite. On 29 July 2021, she increased a methimazole dose from 2.5 to $5 \mathrm{mg}$ daily by herself. Her thyrotoxic symptoms were improved. TFTs at 1 month after an adjusted methimazole dose remained T3-toxicosis with elevated TRAb (Fig. 1). At the 28th day after the dose of

Chutintorn Sriphrapradang

chutins@gmail.com

1 Department of Medicine, Faculty of Medicine Ramathibodi Hospital, Mahidol University, Bangkok 10400, Thailand
ChAdOx1 nCoV-19 vaccine, serum SARS-Cov-2 anti-RBD IgG level was $1991.8 \mathrm{BAU} / \mathrm{mL}$.

The COVID-19 pandemic is now responsible for one of the most challenging and deadliest pandemics. Despite the social distance, mask-wearing, and lockdown, the infected cases remain growing. A mass vaccination campaign is essential to control the COVID-19 pandemic. The mRNA vaccines have shown high levels of efficacy but can activate several proinflammatory pathways that may trigger autoimmune diseases. Heterologous prime-boost vaccination has been recommended as a strategy against emerging SAR-CoV-2 variants. Mixing two different vaccine platforms elicits a stronger immune response than a single vaccine regimen. Heterologous prime-boost with two doses of inactivated vaccine followed by adenovirus-vectored vaccine improves humoral and cellular responses in mice [3], but no human data have been published in a peer-reviewed journal. Currently, an association between Graves'

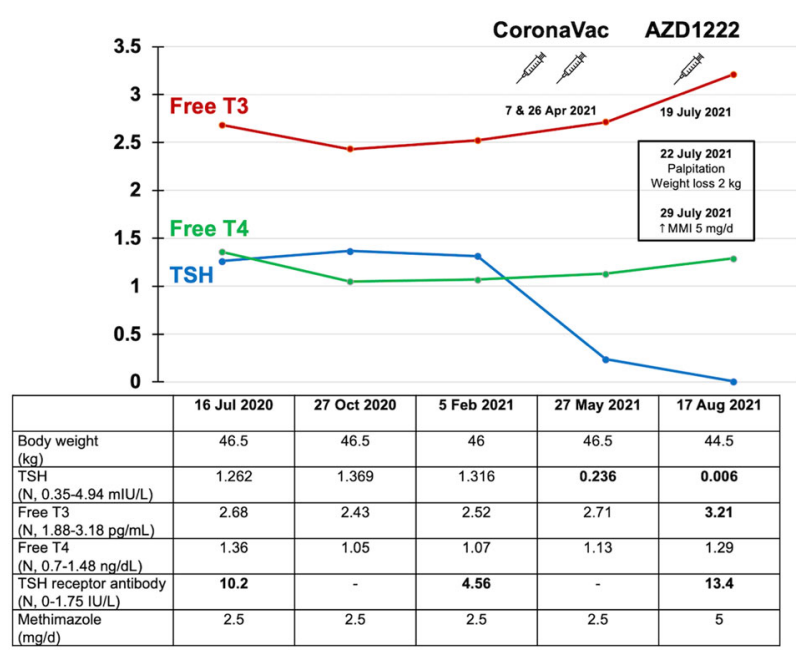

Fig. 1 Summary of thyroid function tests 
disease and the high efficacy SARS-CoV-2 vaccine is still unclear. It is possibly due to an autoimmune/ inflammatory syndrome induced by adjuvants (ASIA) [1]. Physicians should raise awareness regarding the potential for Graves' disease as an adverse effect in order to provoke more observations and promote better understanding. However, vaccine hesitancy should be avoided because Graves' disease is not difficult to treat and the benefit of vaccine protection against COVID-19 is far more significant.

\section{Compliance with ethical standards}

Conflict of interest The author declares no competing interests.

Ethical approval Written consent was obtained from the patient. This case report was approved by the Human Research Ethics Committee, Faculty of Medicine Ramathibodi Hospital, Mahidol University.
Publisher's note Springer Nature remains neutral with regard to jurisdictional claims in published maps and institutional affiliations.

\section{References}

1. O. Vera-Lastra, A. Ordinola Navarro, M.P. Cruz Domiguez, G. Medina, T.I. Sanchez Valadez, L.J. Jara, Two Cases of Graves' Disease Following SARS-CoV-2 Vaccination: an autoimmune/ inflammatory syndrome induced by adjuvants. Thyroid (2021). https://doi.org/10.1089/thy.2021.0142.

2. G. Zettinig, M. Krebs, Two further cases of Graves' disease following SARS-Cov-2 vaccination. J. Endocrinol. Invest. (2021). https://doi.org/10.1007/s40618-021-01650-0.

3. J. Zhang, Q. He, C. An, Q. Mao, F. Gao, L. Bian, X. Wu, Q. Wang, P. Liu, L. Song, Y. Huo, S. Liu, X. Yan, J. Yang, B. Cui, C. Li, J. Wang, Z. Liang, M. Xu, Boosting with heterologous vaccines effectively improves protective immune responses of the inactivated SARS-CoV-2 vaccine. Emerg. Microbes Infect. 10(1), 1598-1608 (2021). https://doi.org/10.1080/22221751.2021.1957401. 